\title{
Photon structure functions and azimuthal correlations of lepton pairs in tagged $\gamma \gamma$ collisions
}

\section{L3 Collaboration}

M. Acciarri ${ }^{\text {aa }}$, O. Adriani ${ }^{\text {p }}$, M. Aguilar-Benitez ${ }^{\mathrm{z}}$, S. Ahlen ${ }^{\mathrm{k}}$, J. Alcaraz ${ }^{\mathrm{z}}$, G. Alemanni v, J. Allaby ${ }^{q}$, A. Aloisio ${ }^{\text {ac }}$, M.G. Alviggi ${ }^{\text {ac }}$, G. Ambrosi ${ }^{\text {s, }}$, H. Anderhub av , V.P. Andreev ak, T. Angelescu ${ }^{\text {m }}$, F. Anselmo ', A. Arefiev ${ }^{\text {ab }}$, T. Azemoon ${ }^{c}$, T. Aziz ${ }^{j}$, P. Bagnaia ${ }^{\text {aj }}$, L. Baksay ${ }^{\text {aq }}$, R.C. Ball ${ }^{c}$, S. Banerjee ${ }^{j}$, Sw. Banerjee ${ }^{j}, K_{\text {. Banicz }}{ }^{\text {as }}$, A. Barczyk ${ }^{\text {av,at }}$, R. Barillère ${ }^{q}$, L. Barone ${ }^{\text {aj, }}$, P. Bartalini v , A. Baschirotto ${ }^{\text {aa }}$, M. Basile ${ }^{\mathrm{i}}$, R. Battiston ${ }^{\mathrm{ag}}$, A. Bay ${ }^{\mathrm{v}}$, F. Becattini ${ }^{\mathrm{p}}$, U. Becker ${ }^{\circ}$, F. Behner av, J. Berdugo ${ }^{\mathrm{z}}$, P. Berges ${ }^{\circ}$, B. Bertucci ${ }^{\text {ag }}$, B.L. Betev ${ }^{\text {av }}$, S. Bhattacharya ${ }^{j}$, M. Biasini ${ }^{\text {ag }}$, A. Biland ${ }^{\text {av }}$, G.M. Bilei ${ }^{\text {ag }}$, J.J. Blaising ${ }^{d}$, S.C. Blyth ${ }^{\text {ah }}$, G.J. Bobbink ${ }^{\mathrm{b}}$, R. Bock ${ }^{\mathrm{a}}$, A. Böhm ${ }^{\mathrm{a}}$, L. Boldizsar ${ }^{\mathrm{n}}$, B. Borgia ${ }^{\text {q,aj, }}$

D. Bourilkov ${ }^{\text {av }}$, M. Bourquin s , D. Boutigny ${ }^{\mathrm{d}}$, S. Braccini ${ }^{\mathrm{s}}$, J.G. Branson ${ }^{\text {am }}$, V. Brigljevic ${ }^{\text {av }}$, I.C. Brock ${ }^{\text {ah }}$, A. Buffini ${ }^{\text {p }}$, A. Buijs ${ }^{\text {ar }}$, J.D. Burger ${ }^{\circ}$, W.J. Burger ${ }^{\text {ag }}$, J. Busenitz ${ }^{\text {aq }}$, X.D. Cai ${ }^{\circ}$, M. Campanelli ${ }^{\text {av }}$, M. Capell ${ }^{\circ}$, G. Cara Romeo ${ }^{\mathrm{i}}$, G. Carlino ${ }^{\text {ac }}$, A.M. Cartacci ${ }^{\text {p }}$, J. Casaus ${ }^{\text {z }}$, G. Castellini ${ }^{\text {p }}$, F. Cavallari aj, N. Cavallo ${ }^{\text {ac }}$, C. Cecchi ${ }^{\mathrm{s}}$, M. Cerrada ${ }^{\mathrm{z}}$, F. Cesaroni ${ }^{\text {w }}$, M. Chamizo ${ }^{\mathrm{z}}$, Y.H. Chang ${ }^{\text {ax }}$, U.K. Chaturvedi ${ }^{\text {r }, ~ S . V . ~ C h e k a n o v ~}{ }^{\text {ae }}$, M. Chemarin ${ }^{\mathrm{y}}$, A. Chen ${ }^{\mathrm{ax}}$, G. Chen ${ }^{\mathrm{g}}$, G.M. Chen ${ }^{\mathrm{g}}$, H.F. Chen ${ }^{\mathrm{t}}$, H.S. Chen ${ }^{\mathrm{g}}$, M. Chen ${ }^{\circ}$, G. Chiefari ${ }^{\mathrm{ac}}$, C.Y. Chien ${ }^{\mathrm{e}}$, L. Cifarelli al , F. Cindolo ${ }^{\mathrm{i}}$, C. Civinini ${ }^{\mathrm{p}}$, I. Clare ${ }^{\circ}$, R. Clare ${ }^{\circ}$, G. Coignet ${ }^{\mathrm{d}}$, A.P. Colijn ${ }^{\mathrm{b}}$, N. Colino ${ }^{\mathrm{z}}$, S. Costantini ${ }^{\mathrm{h}}$, F. Cotorobai ${ }^{\mathrm{m}}$, B. de la $\mathrm{Cruz}^{\mathrm{z}}$, A. Csilling ", T.S. Dai ${ }^{0}$, R. D'Alessandro ${ }^{\mathrm{p}}$, R. de Asmundis ${ }^{\mathrm{ac}}$, A. Degré ${ }^{d}$, K. Deiters ${ }^{\text {at }}$, P. Denes ${ }^{\text {ai }}$, F. DeNotaristefani aj, M. Diemoz aj, D. van Dierendonck ${ }^{\text {b }}$, F. Di Lodovico ${ }^{\text {av }}$, C. Dionisi ${ }^{\text {q.aj }}$, M. Dittmar ${ }^{\text {av }}$, A. Dominguez ${ }^{\text {am }}$, A. Doria ${ }^{\text {ac }}$, M.T. Dova ${ }^{\mathrm{r}, 1}$, E. Drago ${ }^{\text {ac }}$, D. Duchesneau ${ }^{\text {d }}$, P. Duinker ${ }^{\text {b }}$, I. Duran ${ }^{\text {an }}$, S. Easo ${ }^{\text {ag }}$, H. El Mamouni ${ }^{y}$, A. Engler ${ }^{\text {ah }}$, F.J. Eppling ${ }^{o}$, F.C. Erné ${ }^{b}$, J.P. Ernenwein ${ }^{y}$, P. Extermann ${ }^{s}$, M. Fabre ${ }^{\text {at }}$, R. Faccini aj, M.A. Falagan ${ }^{\text {z }}$, S. Falciano ${ }^{\text {aj }}$, A. Favara ${ }^{\text {p }}$, J. Fay ${ }^{y}$, O. Fedin ${ }^{\text {ak }}$, M. Felcini ${ }^{\text {av }}$, T. Ferguson ${ }^{\text {ah }}$, F. Ferroni aj, H. Fesefeldt ${ }^{\text {a }}$, E. Fiandrini ${ }^{\text {ag }}$, J.H. Field ${ }^{\text {s }}$, F. Filthaut ${ }^{\mathrm{q}}$, P.H. Fisher ${ }^{\circ}$, I. Fisk ${ }^{\text {am }}$, G. Forconi ${ }^{\circ}$, L. Fredj ${ }^{\text {, }}$, K. Freudenreich ${ }^{\text {av }}$, C. Furetta ${ }^{\text {aa }}$, 
Yu. Galaktionov ab,o , S.N. Ganguli ${ }^{j}$, P. Garcia-Abia ${ }^{\text {f }}$, M. Gataullin ${ }^{\text {af }}$, S.S. Gau ${ }^{1}$, S. Gentile ${ }^{\text {aj }}$, J. Gerald ${ }^{\mathrm{e}}$, N. Gheordanescu ${ }^{\mathrm{m}}$, S. Giagu ${ }^{\text {aj }}$, S. Goldfarb ${ }^{\mathrm{v}}$, J. Goldstein k , Z.F. Gong ', A. Gougas e, G. Gratta af, M.W. Gruenewald ${ }^{\text {h }}$, R. van Gulik ${ }^{\text {b }}$, V.K. Gupta ${ }^{\text {ai }}$, A. Gurtu ${ }^{j}$, L.J. Gutay ${ }^{\text {as }}$, D. Haas ${ }^{\text {f }}$, B. Hartmann ${ }^{\text {a }}$, A. Hasan ${ }^{\text {ad }}$, D. Hatzifotiadou ${ }^{\text {i }, ~ T . ~ H e b b e k e r ~}{ }^{\text {h }}$, A. Hervé ${ }^{\mathrm{q}}$, P. Hidas ${ }^{n}$, J. Hirschfelder ${ }^{\text {ah }}$, W.C. van Hoek ${ }^{\text {ae }}$, H. Hofer ${ }^{\text {av }}$, H. Hoorani ah, S.R. Hou ${ }^{\text {ax }}$, G. Hu ${ }^{\text {e }}$, I. Iashvili au, B.N. Jin ${ }^{\mathrm{g}}$, L.W. Jones ${ }^{c}$, P. de Jong ${ }^{\mathrm{q}}$, I. Josa-Mutuberria ${ }^{\text {z }}$, A. Kasser ${ }^{\text {v }}$, R.A. Khan ${ }^{r}$, D. Kamrad ${ }^{\text {au }}$, J.S. Kapustinsky ${ }^{x}$, Y. Karyotakis ${ }^{\text {d }}$, M. Kaur ${ }^{\text {r,2 }}$, M.N. Kienzle-Focacci ${ }^{\text {s }}$, D. Kim ${ }^{\text {aj }}$, D.H. Kim ${ }^{\text {ap }}$,

J.K. Kim ${ }^{\text {ap }}$, S.C. Kim ${ }^{\text {ap }}$, W.W. Kinnison ${ }^{x}$, A. Kirkby ${ }^{\text {af }}$, D. Kirkby ${ }^{\text {af }}$, J. Kirkby ${ }^{\text {q }}$, D. Kiss ${ }^{\text {n }}$, W. Kittel ${ }^{\text {ae }}$, A. Klimentov ${ }^{\text {o,ab }}$, A.C. König ${ }^{\text {ae }}$, A. Kopp ${ }^{\text {au }}$, I. Korolko ${ }^{\mathrm{ab}}$, V. Koutsenko ${ }^{\mathrm{o}, \mathrm{ab}}$, R.W. Kraemer ${ }^{\text {ah }}$, W. Krenz ${ }^{\mathrm{a}}$, A. Kunin ${ }^{\text {o,ab }}$,

P. Lacentre ${ }^{a u, 1,3}$, P. Ladron de Guevara ${ }^{z}, G$. Landi ${ }^{p}$, C. Lapoint ${ }^{\circ}$, K. Lassila-Perini ${ }^{\text {av }}$, P. Laurikainen " ${ }^{\text {, A. Lavorato }}{ }^{\text {al }}$, M. Lebeau ${ }^{\mathrm{q}}$, A. Lebedev ${ }^{\circ}$, P. Lebrun ${ }^{y}$, P. Lecomte ${ }^{\text {av }}$, P. Lecoq ${ }^{\mathrm{q}}$, P. Le Coultre ${ }^{\text {av }}$, H.J. Lee ${ }^{\mathrm{h}}$, C. Leggett ${ }^{\mathrm{c}}$, J.M. Le Goff ${ }^{\mathrm{q}}$, R. Leiste ${ }^{\mathrm{au}}$, E. Leonardi ${ }^{\mathrm{aj}}$, P. Levtchenko ${ }^{\mathrm{ak}}, \mathrm{C} \mathrm{Li}^{\mathrm{t}}$, C.H. Lin ${ }^{\text {ax }}$, W.T. Lin ${ }^{a x}$, F.L. Linde ${ }^{b, q}$, L. Lista ${ }^{a c}$, Z.A. Liu ${ }^{\text {g }}$, W. Lohmann ${ }^{\text {au }}$, E. Longo ${ }^{\text {aj }}$, W. Lu ${ }^{\text {af }}$, Y.S. Lu ${ }^{\text {g }}$, K. Lübelsmeyer a , C. Luci ${ }^{\text {qaj }}$, D. Luckey ${ }^{\circ}$, L. Luminari ${ }^{\text {aj }}$, W. Lustermann ${ }^{\text {av }}$, W.G. Ma ${ }^{\mathrm{t}}$, M. Maity ${ }^{\mathrm{j}}$, G. Majumder ${ }^{\mathrm{j}}$, L. Malgeri ${ }^{\mathrm{q}}$, A. Malinin ${ }^{\text {ab }}$, C. Maña ${ }^{\text {z }}$, D. Mangeol ae ${ }^{\text {a }}$ P. Marchesini ${ }^{\text {av }}$, G. Marian ${ }^{\text {aq }, 4}$, A. Marin ${ }^{\text {k}}$, J.P. Martin ${ }^{\text {y }}$, F. Marzano aj, G.G.G. Massaro b , K. Mazumdar ${ }^{\mathrm{j}}$, S. Mele ${ }^{\mathrm{q}}$, L. Merola ${ }^{\mathrm{ac}}$, M. Meschini ${ }^{\mathrm{p}}$, W.J. Metzger ${ }^{\text {ae }}$, M. von der Mey ${ }^{\mathrm{a}}$, Y. Mi ${ }^{\text {}}$, D. Migani ${ }^{\text {i }}$, A. Mihul ${ }^{\mathrm{m}}$, A.J.W. van Mil ${ }^{\text {ae }}$, H. Milcent ${ }^{\mathrm{q}}$, G. Mirabelli aj, J. Mnich ${ }^{\mathrm{q}}$, P. Molnar h, B. Monteleoni p, R. Moore ${ }^{\mathrm{c}}$, T. Moulik ${ }^{\mathrm{j}}$, R. Mount af ${ }^{\text {, }}$ F. Muheim ${ }^{\text {s }}$, A.J.M. Muijs ${ }^{\text {b }}$, S. Nahn ${ }^{\circ}$, M. Napolitano ${ }^{\text {ac }}$, F. Nessi-Tedaldi av, H. Newman ${ }^{\text {af }}$, T. Niessen ${ }^{\text {a }}$, A. Nippe ${ }^{\text {v }}$, A. Nisati ${ }^{\text {aj, H. Nowak }}{ }^{\text {au }}$, Y.D. Oh ${ }^{\text {ap }}$, G. Organtini aj, R. Ostonen ", S. Palit ${ }^{\text {l }}$, C. Palomares ${ }^{z}$, D. Pandoulas a , S. Paoletti aj, P. Paolucci ac, H.K. Park ${ }^{\text {ah }}$, I.H. Park ${ }^{\text {ap }}$, G. Pascale ${ }^{\text {aj }}$, G. Passaleva ${ }^{q}$, S. Patricelli ${ }^{\text {ac }}$, T. Paul ${ }^{1}$, M. Pauluzzi ${ }^{\text {ag }}$, C. Paus ${ }^{\text {q }}$, F. Pauss ${ }^{\text {av }}$, D. Peach ${ }^{\text {q }}$, Y.J. Pei ${ }^{\text {a }}$, S. Pensotti ${ }^{\text {aa }}$, D. Perret-Gallix ${ }^{\mathrm{d}}, \mathrm{B}$. Petersen ${ }^{\text {ae }}, \mathrm{S}$. Petrak ${ }^{\mathrm{h}}$, A. Pevsner ${ }^{\mathrm{e}}$, D. Piccolo ${ }^{\text {ac }}$, M. Pieri ${ }^{\mathrm{p}}$, P.A. Piroué ai, E. Pistolesi ${ }^{\text {aa }}$, V. Plyaskin ${ }^{\mathrm{ab}}, \mathrm{M}$. Pohl ${ }^{\text {av }}$, V. Pojidaev ab,p, H. Postema ${ }^{\circ}$, J. Pothier ${ }^{\mathrm{q}}$, N. Produit s, D. Prokofiev ak, J. Quartieri al, G. Rahal-Callot ${ }^{\text {av }}$, N. Raja ${ }^{\text {j, P.G. Rancoita }}{ }^{\text {aa }}$, M. Rattaggi ${ }^{\text {aa }}$, G. Raven ${ }^{\text {am }}$, P. Razis ${ }^{\text {ad }}$, D. Ren ${ }^{\text {av }}$, M. Rescigno ${ }^{\text {aj }}$, S. Reucroft ${ }^{1}$, T. van Rhee ${ }^{\text {ar }}$, S. Riemann ${ }^{\text {au }}$, K. Riles ${ }^{\text {c }}$, O. Rind ${ }^{\mathrm{c}}$, A. Robohm ${ }^{\text {av }}$, J. Rodin ${ }^{\text {aq }}$, B.P. Roe ${ }^{\mathrm{c}}$, L. Romero $^{z}$, S. Rosier-Lees ${ }^{\text {d, Ph. Rosselet }}$, S. Roth ${ }^{\text {a }}$, J.A. Rubio ${ }^{\mathrm{q}}$, D. Ruschmeier ${ }^{\text {h }}$, H. Rykaczewski ${ }^{\text {av }}$, J. Salicio ${ }^{q}$, E. Sanchez ${ }^{z}$, M.P. Sanders ${ }^{\text {ae }}$, M.E. Sarakinos ", G. Sauvage d, C. Schäfer a, V. Schegelsky ${ }^{\text {ak }}$, 
S. Schmidt-Kaerst a , D. Schmitz ${ }^{\text {a }}$, M. Schneegans ${ }^{\text {d }}$, N. Scholz ${ }^{\text {av }}$, H. Schopper ${ }^{\text {aw }}$, D.J. Schotanus ${ }^{\text {ae }}$, J. Schwenke ${ }^{\text {a }}$, G. Schwering a , C. Sciacca ${ }^{\text {ac }}$, D. Sciarrino s, L. Servoli ag, S. Shevchenko af, N. Shivarov ${ }^{\text {ao }}$, V. Shoutko ${ }^{\text {ab }}$, J. Shukla ${ }^{x}$, E. Shumilov ${ }^{\text {ab }}$, A. Shvorob af, T. Siedenburg ${ }^{\text {a }}$, D. Son ap, V. Soulimov ${ }^{\text {ac }}$, B. Smith ${ }^{\circ}$, P. Spillantini ${ }^{p}$, M. Steuer ${ }^{\circ}$, D.P. Stickland ${ }^{\text {ai }}$, H. Stone ${ }^{\text {ai }}$, B. Stoyanov ${ }^{\text {ao }}$, A. Straessner a , K. Sudhakar ${ }^{\text {j, G. Sultanov }}{ }^{\text {r, }}$, L.Z. Sun ${ }^{\text {t }}$, G.F. Susinno ${ }^{\text {s, H. Suter }}{ }^{\text {av }}$, J.D. Swain ${ }^{\mathrm{r}}$, X.W. Tang ${ }^{\mathrm{g}}$, L. Tauscher ${ }^{\mathrm{f}}$, L. Taylor ${ }^{1}$, Samuel C.C. Ting ${ }^{\circ}$, S.M. Ting ${ }^{\circ}$, S.C. Tonwar ${ }^{\mathrm{j}}$, J. Tóth ${ }^{\mathrm{n}}$, C. Tully ai,

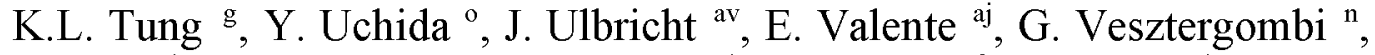
I. Vetlitsky ${ }^{\mathrm{ab}}$, G. Viertel ${ }^{\text {av }}$, M. Vivargent ${ }^{\mathrm{d}}$, S. Vlachos ${ }^{\mathrm{f}}, \mathrm{H}$. Vogel ${ }^{\text {ah }}, \mathrm{H}_{\text {. Vogt }}{ }^{\text {au }}$, I. Vorobiev ${ }^{\text {qab }}$, A.A. Vorobyov ${ }^{a k}$, A. Vorvolakos ${ }^{a d}$, M. Wadhwa ${ }^{f}$, W. Wallraff a J.C. Wang ${ }^{\circ}$, X.L. Wang ${ }^{\mathrm{t}}$, Z.M. Wang ${ }^{\mathrm{t}}$, A. Weber ${ }^{\mathrm{a}}$, S.X. Wu ${ }^{\circ}$, S. Wynhoff ${ }^{\mathrm{a}}$, J. Xu ${ }^{k}$, Z.Z. Xu ${ }^{\mathrm{t}}$, B.Z. Yang ${ }^{\mathrm{t}}$, C.G. Yang ${ }^{\mathrm{g}}$, H.J. Yang ${ }^{\mathrm{g}}$, M. Yang ${ }^{\mathrm{g}}$, J.B. Ye ${ }^{\mathrm{t}}$, S.C. Yeh ay , J.M. You ${ }^{\text {ah }}$, An. Zalite ${ }^{\text {ak }}$, Yu. Zalite ${ }^{\text {ak }}$, P. Zemp ${ }^{\text {av }}$, Y. Zeng ${ }^{\text {a }}$, Z.P. Zhang ${ }^{\text {t, B. Zhou }}{ }^{\text {k, }}$ Y. Zhou ${ }^{\text {c }}$, G.Y. Zhu ${ }^{\text {g }}$, R.Y. Zhu ${ }^{\text {af }}$, A. Zichichi ${ }^{\text {i, }, \text {, }}$, F. Ziegler ${ }^{\text {au }}$, G. Zilizi aq,4

\footnotetext{
${ }^{a}$ I. Physikalisches Institut, RWTH, D-52056 Aachen, FRG, and III. Physikalisches Institut, RWTH, D-52056 Aachen, FRG ${ }^{5}$ ${ }^{\mathrm{b}}$ National Institute for High Energy Physics, NIKHEF, and University of Amsterdam, NL-1009 DB Amsterdam, The Netherlands ' University of Michigan, Ann Arbor, MI 48109, USA

'Laboratoire d'Annecy-le-Vieux de Physique des Particules, LAPP,IN2P3-CNRS, BP 110, F-74941 Annecy-le-Vieux CEDEX, France

' Johns Hopkins University, Baltimore, MD 21218, USA

${ }^{\mathrm{f}}$ Institute of Physics, University of Basel, CH-4056 Basel, Switzerland

${ }^{\mathrm{g}}$ Institute of High Energy Physics, IHEP, 100039 Beijing, China ${ }^{6}$

${ }^{\mathrm{h}}$ Humboldt University, D-10099 Berlin, FRG ${ }^{5}$

${ }^{\mathrm{i}}$ University of Bologna and INFN-Sezione di Bologna, I-40126 Bologna, Italy

${ }^{\mathrm{j}}$ Tata Institute of Fundamental Research, Bombay 400005 , India

${ }^{\mathrm{k}}$ Boston University, Boston, MA 02215, USA

${ }^{1}$ Northeastern University, Boston, MA 02115, USA

${ }^{\mathrm{m}}$ Institute of Atomic Physics and University of Bucharest, R-76900 Bucharest, Romania

${ }^{\mathrm{n}}$ Central Research Institute for Physics of the Hungarian Academy of Sciences, H-1525 Budapest 114, Hungary ${ }^{7}$

${ }^{\circ}$ Massachusetts Institute of Technology, Cambridge, MA 02139, USA

${ }^{\mathrm{p}}$ INFN Sezione di Firenze and University of Florence, I-50125 Florence, Italy

${ }^{\mathrm{q}}$ European Laboratory for Particle Physics, CERN, CH-1211 Geneva 23, Switzerland

${ }^{r}$ World Laboratory, FBLJA Project, CH-1211 Geneva 23, Switzerland

"University of Geneva, $\mathrm{CH}-1211$ Geneva 4, Switzerland

${ }^{\mathrm{t}}$ Chinese University of Science and Technology, USTC, Hefei, Anhui 230 029, China ${ }^{6}$

" SEFT, Research Institute for High Energy Physics, P.O. Box 9, SF-00014 Helsinki, Finland

"University of Lausanne, $\mathrm{CH}-1015$ Lausanne, Switzerland

${ }^{w}$ INFN-Sezione di Lecce and Universitá Degli Studi di Lecce, I-73100 Lecce, Italy

${ }^{x}$ Los Alamos National Laboratory, Los Alamos, NM 87544, USA

y Institut de Physique Nucléaire de Lyon, IN2P 3-CNRS, Université Claude Bernard, F-69622 Villeurbanne, France

${ }^{\mathrm{z}}$ Centro de Investigaciones Energeticas, Medioambientales y Tecnologicas, CIEMAT, E-28040 Madrid, Spain ${ }^{8}$

aa INFN-Sezione di Milano, I-20133 Milan, Italy

ab Institute of Theoretical and Experimental Physics, ITEP, MOscow, Russia

ac INFN-Sezione di Napoli and University of Naples, I-80125 Naples, Italy

ad Department of Natural Sciences, University of Cyprus, Nicosia, Cyprus

${ }^{a}$ University of Nijmegen and NIKHEF, NL-6525 ED Nijmegen, The Netherlands

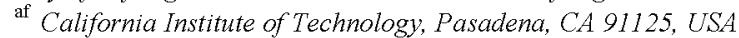

ag INFN-Sezione di Perugia and Universitá Degli Studi di Perugia, I-06100 Perugia, Italy
} 
ah Carnegie Mellon University, Pittsburgh, PA 15213, USA

ai Princeton University, Princeton, NJ 08544, USA

aj INFN-Sezione di Roma and University of Rome, "La Sapienza", I-00185 Rome, Italy

${ }^{\text {ak }}$ Nuclear Physics Institute, St. Petersburg, Russia

al University and INFN, Salerno, I-84100 Salerno, Italy

am University of California, San Diego, CA 92093, USA

an Dept. de Fisica de Particulas Elementales, Univ. de Santiago, E-15706 Santiago de Compostela, Spain

ao Bulgarian Academy of Sciences, Central Lab. of Mechatronics and Instrumentation, BU-1113 Sofia, Bulgaria

ap Center for High Energy Physics, Adv. Inst. of Sciences and Technology, 305-701 Taejon, South Korea

aq University of Alabama. Tuscaloosa, AL 35486, USA

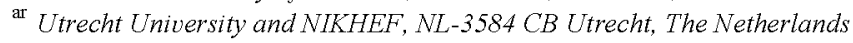

${ }^{\text {as }}$ Purdue University, West Lafayette, IN 47907, USA

${ }^{a t}$ Paul Scherrer Institut, PSI, CH-5232 Villigen, Switzerland

${ }^{\text {au }}$ DESY-Institut fir Hochenergiephysik, D-15738 Zeuthen, FRG

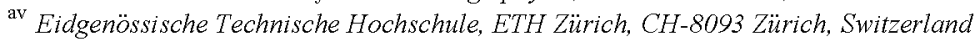

aw University of Hamburg, D-22761 Hamburg, FRG

ax National Central University, Chung-Li, Taiwan, ROC

ay Department of Physics, National Tsing Hua University, Taiwan, China

Received 1 May 1998

Editor: K. Winter

\begin{abstract}
The reactions $\mathrm{e}^{+} \mathrm{e}^{-} \rightarrow \mathrm{e}^{+} \mathrm{e}^{-} \mathrm{e}^{+} \mathrm{e}^{-}$and $\mathrm{e}^{+} \mathrm{e}^{-} \rightarrow \mathrm{e}^{+} \mathrm{e}^{-} \mu^{+} \mu^{-}$, in a single tag configuration, are studied at LEP with the $\mathrm{L} 3$ detector. The data set corresponds to an integrated luminosity of $93.7 \mathrm{pb}^{-1}$ at $\sqrt{\mathrm{s}}=91 \mathrm{GeV}$. Differential cross sections are measured for $1.4 \mathrm{GeV}^{2} \leq Q^{2} \leq 7.6 \mathrm{GeV}^{2}$. The leptonic photon structure function $F_{2}^{\gamma}$ and azimuthal correlations are measured for $\mathrm{e}^{+} \mathrm{e}^{-} \rightarrow \mathrm{e}^{+} \mathrm{e}^{-} \mu^{+} \mu^{-}$. The related structure functions $F_{A}^{\gamma}$ and $F_{B}^{\gamma}$, which originate from interference terms of the scattering amplitudes, are determined for the first time. (C) 1998 Published by Elsevier Science B.V. All rights reserved.
\end{abstract}

\title{
1. Introduction
}

The reaction $\mathrm{e}^{+} \mathrm{e}^{-} \rightarrow \mathrm{e}^{+} \mathrm{e}^{-} l^{+} l^{-}$, where $l$ can be any charged lepton, arises from $O\left(\alpha^{4}\right)$ QED processes [1] Usually the scattered electrons are undetected; the analysis of this configuration has been published in Ref. [2]. In this paper we study the single tag configuration, where one of the scattered electrons is measured. This reaction is treated as an electron-photon deep inelastic scattering process; it provides not only a test of QED but also a check of the experimental procedures adopted in the analysis of the hadronic photon structure functions.

\footnotetext{
${ }^{1}$ Also supported by CONICET and Universidad Nacional de La Plata, CC 67, 1900 La Plata, Argentina.

${ }^{2}$ Also supported by Panjab University, Chandigarh-160014, India.

${ }^{3}$ Supported by Deutscher Akademischer Austauschdienst.

${ }^{4}$ Also supported by the Hungarian OTKA fund under contract numbers T22238 and T026178.

${ }^{5}$ Supported by the German Bundesministerium für Bildung, Wissenschaft, Forschung und Technologie.

${ }^{6}$ Supported by the National Natural Science Foundation of China.

${ }^{7}$ Supported by the Hungarian OTKA fund under contract numbers T019181, F023259 and T024011.

${ }^{8}$ Supported also by the Comisión Interministerial de Ciencia y Technología.
} 
Using data collected from 1991 to 1994 with the L3 detector at LEP, with a beam energy $E_{\text {beam }} \simeq 45.6 \mathrm{GeV}$, we investigate the reactions:

$$
\begin{aligned}
& \text { e } \gamma^{*} \rightarrow \mathrm{e} \mathrm{e}^{+} \mathrm{e}^{-}, \\
& \text {e } \gamma^{*} \rightarrow \mathrm{e} \mu^{+} \mu^{-},
\end{aligned}
$$

where the three final state leptons are detected. The measurement of the scattered electron determines the four-vector of the radiated virtual photon, which is the probe photon. The target virtual photon, $\gamma^{*}$, radiated by the unobserved electron, is almost real. Three leptonic photon structure functions $F_{2}^{\gamma}, F_{A}^{\gamma}$ and $F_{B}^{\gamma}$, related to different helicity states of the colliding photons, are extracted from the measured cross sections for e $\gamma^{*} \rightarrow$ e $\mu^{+} \mu^{-}$. At LEP, the leptonic structure function $F_{2}^{\gamma}$ has been measured previously $[3,4]$ and the ratio $F_{B}^{\gamma} / F_{2}^{\gamma}$ was extracted from azimuthal correlations [5].

\section{Formalism}

The lowest order Feynman diagrams which describe the reactions $\mathrm{e}^{+} \mathrm{e}^{-} \rightarrow \mathrm{e}^{+} \mathrm{e}^{-} l^{+} l^{-}$involve several processes classified as multiperipheral, annihilation, bremsstrahlung and conversion [2]. The dominant contribution is the multiperipheral diagram, which includes the interaction of two virtual photons. Annihilation and bremsstrahlung contributions become important only for small effective masses of the produced lepton pair. The incoming beam electrons, with four-momenta $p_{1}, p_{2}$, are scattered through polar angles $\theta_{1}, \theta_{2}$ with energies $E_{1}, E_{2}$ respectively. The probe and target photon have four-momenta $k_{1}=\left(x_{1} E_{\text {beam }}, \boldsymbol{k}_{1}\right)$ and $k_{2}=\left(x_{2} E_{\text {beam }}, \boldsymbol{k}_{2}\right)$ and virtualities $Q^{2}=-k_{1}^{2}$ and $t^{2}=-k_{2}^{2}$, where:

$$
Q^{2} \simeq 2 E_{\text {beam }} E_{1}\left(1-\cos \theta_{1}\right) \text {. }
$$

The Bjorken scaling variables $x$ and $y$ are defined as:

$$
x=\frac{Q^{2}}{2 k_{1} \cdot k_{2}}=\frac{Q^{2}}{Q^{2}+t^{2}+W_{\gamma \gamma}^{2}}, \quad y=\frac{k_{2} \cdot k_{1}}{k_{2} \cdot p_{1}} \simeq 1-\frac{E_{1}}{E_{\text {beam }}} \cos ^{2} \frac{\theta_{1}}{2} \simeq x_{1},
$$

where the mass squared of the produced lepton pair is $M_{l l}^{2}=W_{\gamma \gamma}^{2}=\left(k_{1}+k_{2}\right)^{2}$. The cross section for deep-inelastic electron scattering on a photon target is the sum of the contributions of transverse $(T)$ and longitudinal (L) photons. The differential cross section is written as [6]:

$$
\frac{d^{3} \sigma}{d x d Q^{2} d x_{2}}=\frac{4 \pi \alpha^{2}}{Q^{4}} \frac{1}{x} \frac{d n\left(x_{2}\right)}{d x_{2}}\left\{\left(1-y+\frac{y^{2}}{2}\right) F_{2}^{\gamma}\left(x, Q^{2}\right)+\frac{y^{2}}{2} F_{L}^{\gamma}\left(x, Q^{2}\right)\right\},
$$

where the flux of target photons is given by:

$$
\frac{d n\left(x_{2}\right)}{d x_{2}}=\frac{\alpha}{\pi x_{2}}\left\{\left[1+\left(1-x_{2}\right)^{2}\right] \ln \left(\frac{2 E_{\text {beam }}\left(1-x_{2}\right)}{m_{e} x_{2}} \sin \frac{\theta_{2}^{\max }}{2}\right)-1+x_{2}\right\} .
$$

The polar angle $\theta_{2}$ of the unobserved electron is restricted to be smaller than $\theta_{2}^{\text {max }}$ which is also the minimum polar angle for the tagged electron, $\theta_{1}^{\min }$. The photon structure functions $F_{T}^{\gamma}$ and $F_{L}^{\gamma}$ are proportional to the cross sections $\sigma_{i j}$ for transversely and longitudinally polarised virtual photons

$$
F_{T}^{\gamma} \propto \sigma_{T T}, F_{L}^{\gamma} \propto \sigma_{T L} \text { and } F_{2}^{\gamma}=2 x F_{T}^{\gamma}+F_{L}^{\gamma}
$$



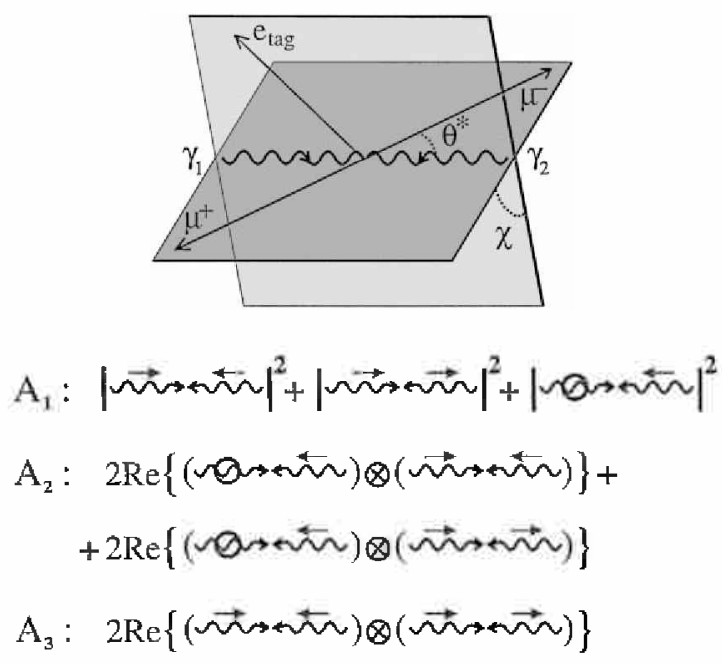

Fig. 1. Definition of the angles $\theta^{*}$ and $\chi$ in the $\gamma \gamma$ centre-of-mass frame. For a single tag configuration, the target photon is almost real and, in good approximation, purely transverse. There are only three possible independent helicity amplitudes. The relation between the coefficients $A_{i}$ and the photon helicity states are schematically shown. $A_{1}$ is the quadratic sum of the total photon helicity $\lambda=0,2$ and 1 while $A_{2}$ and $A_{3}$ are interference terms. Target photons are represented on the right and probe photons on the left. The longitudinal component of the probe photon can be obtained from a measurement of $A_{2}$.

The first index of the cross sections refers to the target photon, the second to the probe photon. In the present analysis, $y^{2}$ is small, on average $<4.5 \cdot 10^{-4}$ : therefore the measurement of the differential cross section is sensitive only to $F_{2}^{\gamma}$. Neglecting terms of order $m_{l}^{2} / Q^{2}$, the QED prediction for the photon structure function $F_{2}^{\gamma}$ is [6]:

$$
F_{2}^{\gamma}=\frac{\alpha}{\pi} x\left\{\left[x^{2}+(1-x)^{2}\right] \ln \frac{W_{\gamma \gamma}^{2}}{m_{l}^{2}+t^{2} x(1-x)}-1+8 x(1-x)+\frac{t^{2} x(1-x)}{m_{l}^{2}+t^{2} x(1-x)}\right\} .
$$

The scattering amplitude of longitudinally polarised photons is observed by measuring angular correlations in the $\gamma \gamma$ centre-of-mass system [6-9]. The polar angle $\theta^{*}$ and the azimuthal angle $\chi$ of the $\mu^{-}$are defined as shown in Fig. 1, where the target photon direction is assumed to be parallel to the beam axis. The azimuthal angle $\chi$ is defined as the angle between the scattering plane of the tagged electron and the plane defined by the $\mu^{-}$direction and the $\gamma \gamma$ axis. The decomposition of the cross section into contributions from the different photon helicity amplitudes leads to a formula with 13 terms, each having a different azimuthal dependence. In the single tag configuration, after neglecting the longitudinal component of the target photon, only three terms remain in the cross section formula:

$$
\frac{d^{4} \sigma}{d x d y d z d \chi}=\frac{2 \pi \alpha^{2}}{Q^{2}} \frac{1+(1-y)^{2}}{x y}\left[A_{1}(x, z)+A_{2}(x, z) \cdot \cos \chi+A_{3}(x) \cdot \cos 2 \chi\right],
$$

where $z=\cos \theta^{*}$. The coefficients $A_{1}, A_{2}$ and $A_{3}$ are related to three differential structure functions $\tilde{F}_{2}^{\gamma}, \tilde{F}_{A}^{\gamma}$ and $\tilde{F}_{B}^{\gamma}[8]$ :

$$
A_{1}=\tilde{F}_{2}^{\gamma}(x, z)=2 x \tilde{F}_{T}^{\gamma}(x, z)+\tilde{F}_{L}^{\gamma}(x), \quad A_{2}=-\tilde{F}_{A}^{\gamma}(x, z), \quad A_{3}=\frac{1}{2} \tilde{F}_{B}^{\gamma}(x) .
$$


The sum of the amplitudes squared of the helicity states is given by $A_{1}$, while $A_{2}$ and $A_{3}$ are interference terms leading to $\cos \chi$ and $\cos 2 \chi$ modulations [7]. The coefficients $A_{i}$ are calculable in QED. Setting $1-y \simeq 1$ in the $\gamma \gamma$ luminosity functions [1] they are expressed by :

$$
\begin{aligned}
& A_{1}=\frac{x \alpha}{\pi}\left(\left[x^{2}+(1-x)^{2}\right] \frac{1+(\beta z)^{2}}{1-(\beta z)^{2}}+4 x(1-x)\right), \\
& A_{2}=-\frac{4 \alpha}{\pi} x(1-2 x) \sqrt{x(1-x)} \frac{z}{\sqrt{1-(\beta z)^{2}}}, \\
& A_{3}=\frac{2 \alpha}{\pi} x^{2}(1-x),
\end{aligned}
$$

where $\beta=\sqrt{1-4 x m_{l}^{2} /\left[Q^{2}(1-x)\right]}$. The structure functions $F_{2}^{\gamma}, F_{A}^{\gamma}$ and $F_{B}^{\gamma}$ are obtained by integrating $\tilde{F}_{i}^{\gamma}$ over $z$. The coefficient $A_{2}$ is sensitive to the amplitude with two-photon helicity $\lambda=1$, see Fig. 1, whose square is the longitudinal structure function. The interference between two transverse photons is described by $A_{3} \propto \tilde{F}_{B}^{\gamma}$. It must be noted that, although $F_{L}^{\gamma}$ and $F_{B}^{\gamma}$ are described in QED by the same function of $x$, and, although in the literature both are referred to as $F_{L}^{\gamma}$ [9], they correspond to a different helicity structure of the two photons. Indeed, in $F_{B}^{\gamma}$ the photons are purely transverse.

\section{Data analysis}

The data have been collected at LEP from 1991 to 1994 at the $Z$ peak and correspond to an integrated luminosity of $93.7 \mathrm{pb}^{-1}$. A description of the L3 detector is given elsewhere [10]. The analysis described in this paper is based on the central tracker, the BGO electromagnetic (EM) and the hadron calorimeters. The luminosity monitors [11], consisting of two BGO electromagnetic calorimeters, allow electron tagging in the regions $1.4^{\circ} \leq \theta \leq 3.9^{\circ}$ and $176.1^{\circ} \leq \theta \leq 178.6^{\circ}$.

Single tag two-photon events are mainly collected by a trigger requiring an energy deposit greater than 30 $\mathrm{GeV}$ in the luminosity monitors and at least one track in the central tracking chamber with a transverse momentum $p_{t}>0.1 \mathrm{GeV}$. Other triggers $[10,12]$ may be activated by the same events and allow to measure the trigger inefficiencies. They are $1.3 \%$ for the electron channel and $2.7 \%$ for the muon channel, independent of $Q^{2}$ and $x$.

The tagged electron candidate is defined as a single shower energy deposit in the luminosity monitors with $E \geq 0.75 E_{\text {beam }}$, within the fiducial regions $1.55^{\circ} \leq \theta \leq 3.67^{\circ}$ and $176.33^{\circ} \leq \theta \leq 178.45^{\circ}$, which excludes the innermost and outermost crystals of the detectors. Single tag events are selected by requiring that no other electron candidate with $E \geq 0.75 E_{\text {beam }}$ is seen in the complete L3 detectors. Double-tag event candidates are a few per cent of the single tag sample and are efficiently rejected.

The lepton pair is selected by requiring two tracks with opposite charges, inside a polar angle region $28^{\circ} \leq \theta \leq 152^{\circ}$, with a maximum distance of closest approach from the nominal vertex, in the plane transverse to the beam direction, of $10 \mathrm{~mm}$ and a transverse momentum $p_{t}>0.1 \mathrm{GeV}$.

Table 1

Number of selected events, after background subtraction, compared to the expectations of [14]

\begin{tabular}{llll}
\hline Process & Observed events & Expected events & Ratio \\
\hline $\mathrm{e}^{+} \mathrm{e}^{-} \mu^{+} \mu^{-}$ & $7152 \pm 85$ & $7119 \pm 42$ & $1.005 \pm 0.013$ \\
$\mathrm{e}^{+} \mathrm{e}^{-} \mathrm{e}^{+} \mathrm{e}^{-}$ & $3802 \pm 62$ & $3842 \pm 47$ & $0.990 \pm 0.020$ \\
\hline
\end{tabular}


The electrons and muons are identified by comparing the momentum measured by the tracking chamber with the energy deposit in the electromagnetic calorimeter, $E_{\mathrm{EM}}$, and by using hadron calorimeter information. Electrons are identified by the following criteria:

- in the central region, $44^{\circ} \leq \theta \leq 136^{\circ}$, the ratio $R=E_{\mathrm{EM}} \sin \theta / p_{t}$ must be greater than 0.8 and the energy deposit $E_{\mathrm{EM}} \geq 0.4 \mathrm{GeV}$

- in the forward-backward regions, where the measurements are less accurate, $R$ must be greater than 0.7 and $E_{\mathrm{EM}} \geq 0.6 \mathrm{GeV}$.

Muons are identified by the following criteria:

- an energy deposit $E_{\mathrm{EM}}<0.40 \mathrm{GeV}$ in the electromagnetic calorimeter, where the signal of a minimum ionising particle is, on average, $0.22 \mathrm{GeV}$. More than $95 \%$ of the energy must be contained in at most 6 neighbouring BGO crystals
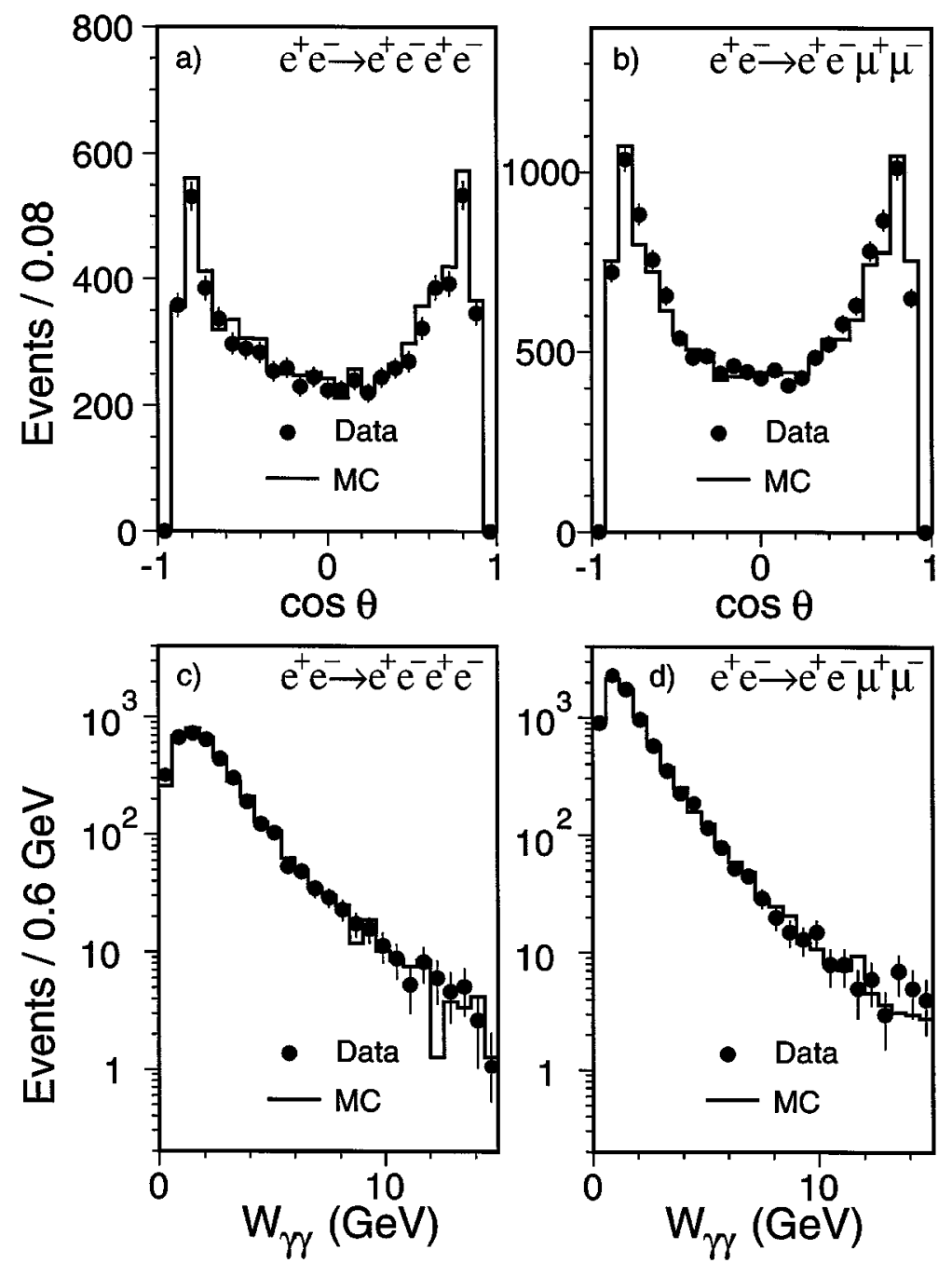

Fig. 2. Comparison of data and Monte Carlo [14], normalized to the integrated luminosity, for the lepton (e or $\mu$ ) polar angle and the two photon mass. The reaction $\mathrm{e}^{+} \mathrm{e}^{-} \rightarrow \mathrm{e}^{+} \mathrm{e}^{-} \mathrm{e}^{+} \mathrm{e}^{-}$is in (a) and (c), the reaction $\mathrm{e}^{+} \mathrm{e}^{-} \rightarrow \mathrm{e}^{+} \mathrm{e}^{-} \mu^{+} \mu^{-}$in (b) and (d). 
- an energy deposit in the hadron calorimeter, consistent with a minimum ionising particle, associated with the selected track. This requirement implies that the energy of the muon is greater than $1.7 \mathrm{GeV}$.

For both $\mathrm{e}^{+} \mathrm{e}^{-}$and $\mu^{+} \mu^{-}$pairs, only one identified particle is required in order to cover uniformly the angular range defined by the tracks. The effective mass of the lepton pair is required to be greater than $0.5 \mathrm{GeV}$.

For the $\mathrm{e}^{+} \mathrm{e}^{-}$sample, in $76 \%$ of the events both electrons are identified. For the $\mu^{+} \mu^{-}$sample, in $40 \%$ of the events both muons reach the hadron calorimeter and have a minimum ionising particle signature. A more detailed description of the analysis is given in Ref. [13].

Two event generators [14,15] together with a full detector simulation [16], are used to calculate the selection efficiencies. The first one [14] calculates the full set of QED diagrams to $O\left(\alpha^{4}\right)$, taking into account interference effects. It shows that, in the kinematical regions of this analysis, only the contribution of the multiperipheral diagrams is non-negligible. The second one [15] which is much faster and has exact calculations only for the multiperipheral diagram, is used to produce large statistics Monte Carlo samples. These generators do not simulate initial state radiation. To estimate the effect of initial state radiation another generator [17], which simulates the process $\mathrm{e}^{+} \mathrm{e}^{-} \rightarrow \mathrm{e}^{+} \mathrm{e}^{-} \mu^{+} \mu^{-}(\gamma)$, is used.

The same generators $[14,15]$ are also used to estimate the $\mathrm{e}^{+} \mathrm{e}^{-} \rightarrow \mathrm{e}^{+} \mathrm{e}^{-} \boldsymbol{\tau}^{+} \tau^{-}$and $\mathrm{e}^{+} \mathrm{e}^{-} \rightarrow \mathrm{e}^{+} \mathrm{e}^{-} q \bar{q}$ backgrounds. The background is $0.9 \%$ for the electron channel dominated by $\tau^{+} \tau^{-}$production. It is $1.8 \%$ for the muon channel due to misidentified electrons $(0.78 \%), \tau^{+} \tau^{-}$production $(0.71 \%)$ and $q \bar{q}$ production $(0.32 \%)$.

The background from radiative $Z$ decays, where an emitted photon fakes the tagged electron, is found to be negligible, by simulating the annihilation processes with the generators KORALZ [18] and JETSET [19].

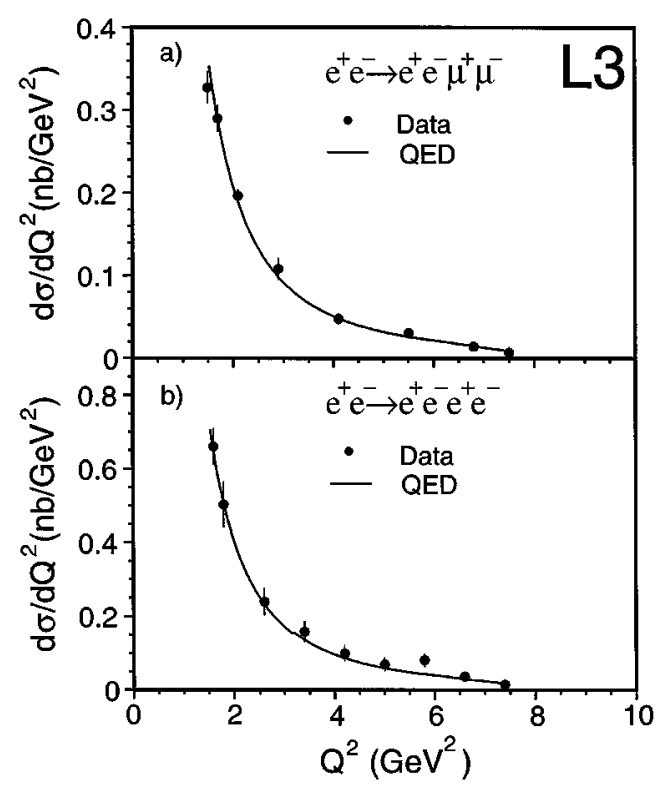

Fig. 3. Differential cross section as function of $Q^{2}$, for $W_{\gamma \gamma} \geq 0.5 \mathrm{GeV}$, for the reactions (a) $\mathrm{e}^{+} \mathrm{e}^{-} \rightarrow \mathrm{e}^{+} \mathrm{e}^{-} \mu^{+} \mu^{-}$and (b) $\mathrm{e}^{+} \mathrm{e}^{-} \rightarrow$ $\mathrm{e}^{+} \mathrm{e}^{-} \mathrm{e}^{+} \mathrm{e}^{-}$. The QED predictions are obtained from Eq. (1) and Eq. (3) using a mean virtuality $<t^{2}>=0.033 \mathrm{GeV}^{2}$ for the target photon. 


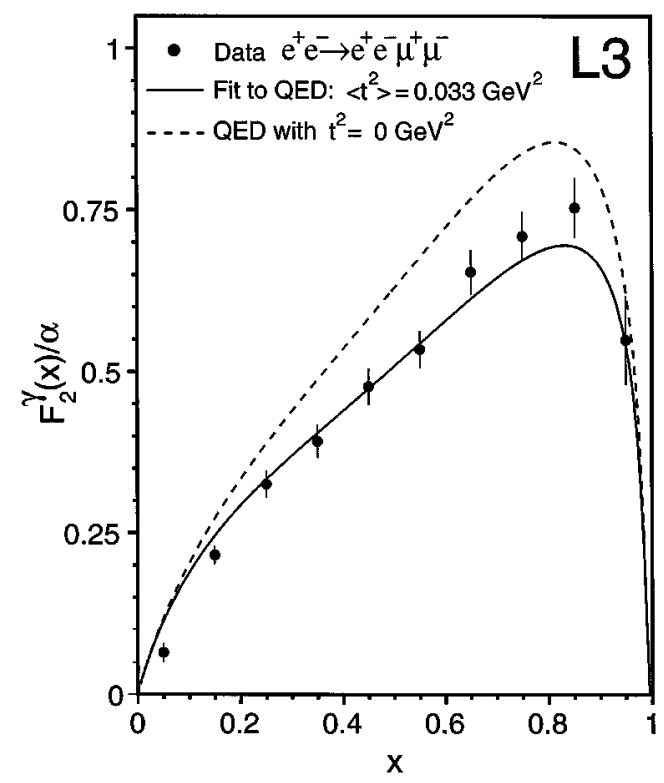

Fig. 4. The leptonic structure function $F_{2}^{\gamma}$ measured in the range $1.4 \mathrm{GeV}^{2} \leq Q^{2} \leq 7.6 \mathrm{GeV}^{2}$. The dashed line corresponds to QED predictions obtained from Eq. (3) with $t^{2}=0$. The solid line is obtained by fitting the mean virtuality of the target photon to the data. Only statistical errors are shown, in addition there is a systematic scale uncertainty of $\pm 3.3 \%$.

The number of selected events, for each channel, after background subtraction, is reported in Table 1 together with the Monte Carlo [14] expectations. The distributions of the polar angle and of the effective mass $W_{\gamma \gamma}$ for the electron and muon pairs are shown in Fig. 2. They are in agreement with the Monte Carlo [14] expectations in shape and in absolute value.

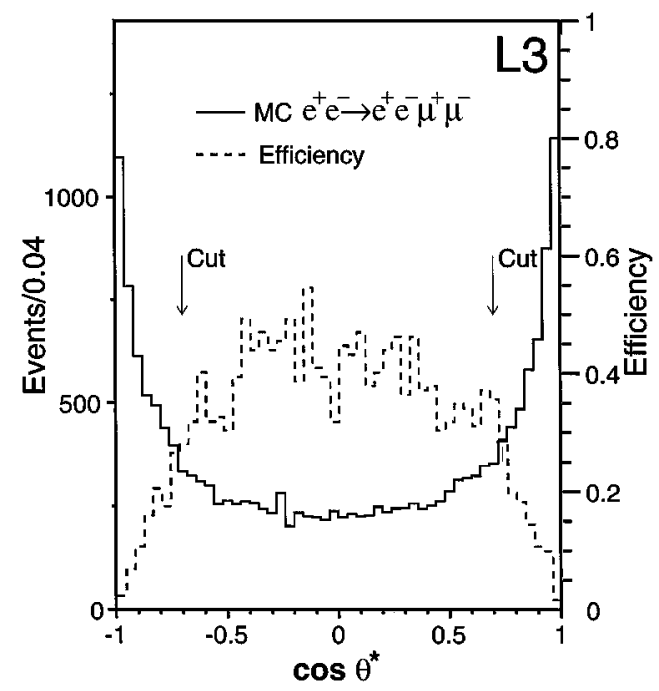

Fig. 5. Monte Carlo angular distribution of $\cos \theta^{*}$ (solid histogram, left scale) and the corresponding selection efficiency (dashed histogram, right scale). The two arrows indicate the region used in the analysis. 


\section{Results}

To calculate the differential cross sections, the data are corrected for acceptance and efficiency in bins of $x$ and $Q^{2}$. The differential cross sections $d \sigma / d Q^{2}$ are shown in Fig. 3 for $1.4 \mathrm{GeV}^{2}<Q^{2}<7.6 \mathrm{GeV}^{2}$ and $W_{\gamma \gamma}>0.5 \mathrm{GeV}$, together with analytical QED predictions obtained from Eq. (1).

The systematic error comes from selection procedure, trigger efficiency, background subtraction and luminosity measurements. It is $3.3 \%$ for the di-muon channel and $1.7 \%$ for the di-electron channel and is found to be independent of $Q^{2}$ and $x$ [13].
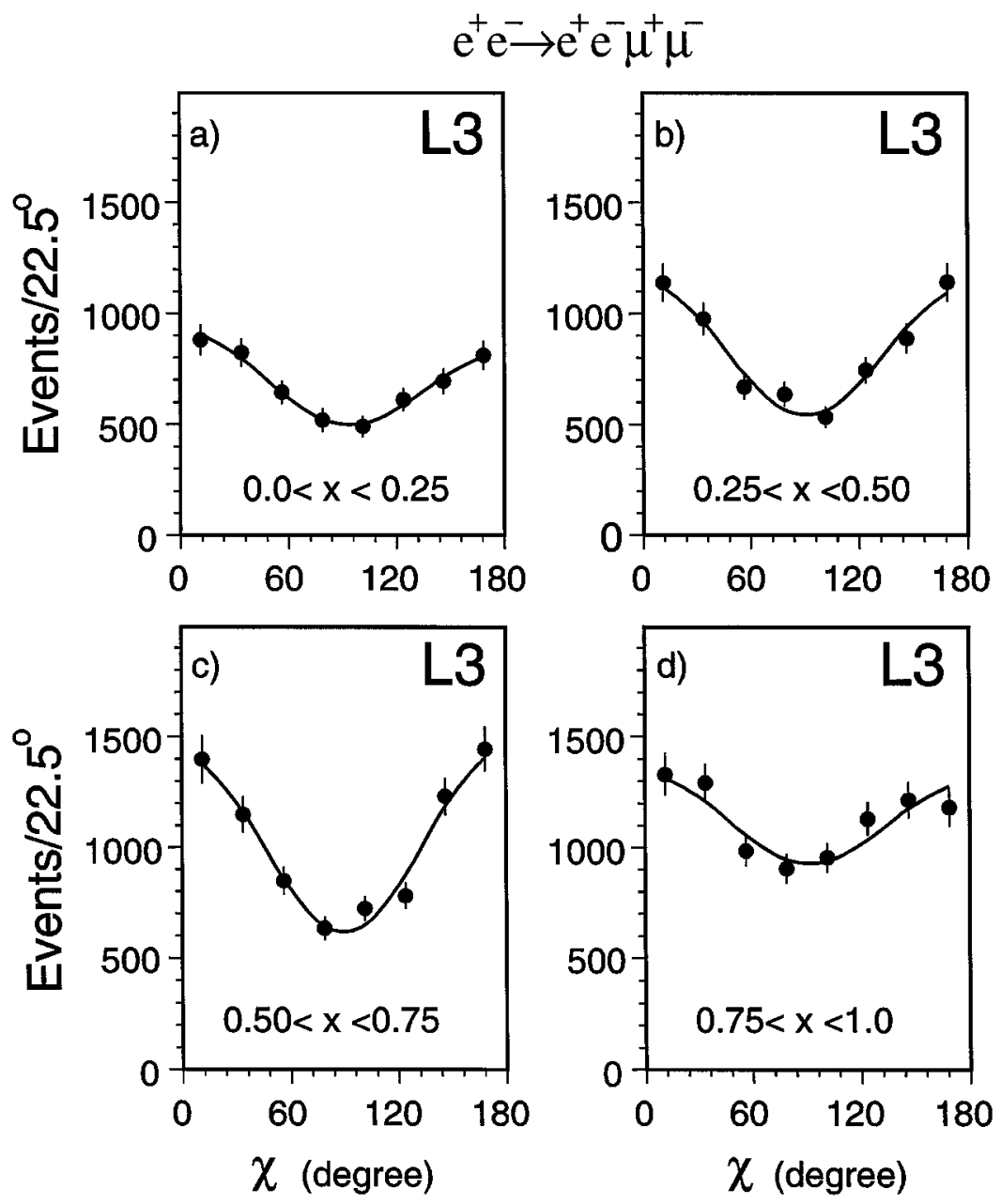

Fig. 6. The $\mu^{-}$azimuthal angle distribution for $|z|<0.7$ in four intervals of $x$. The data are corrected for selection efficiency. The curves show the function given in Eq. (7) with the value of $R_{2 \chi}$ determined by a fit to the data in each $x$ interval (see Table 2 ). 


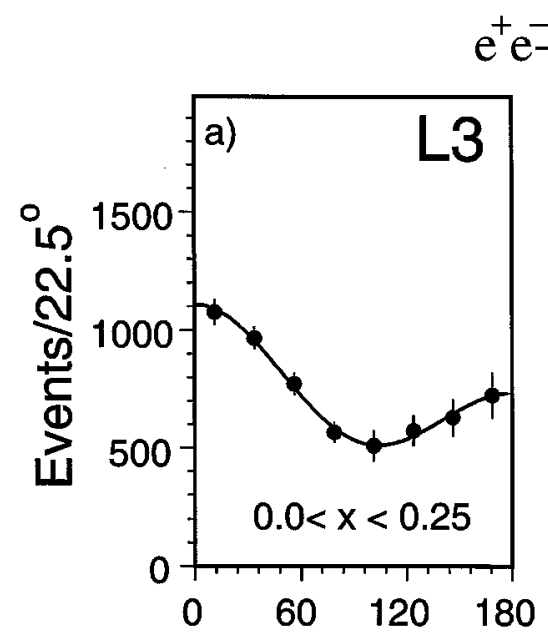

$\mathrm{e}^{+} \mathrm{e} \rightarrow \mathrm{e}^{+} \mathrm{e}^{-} \mu^{+} \mu^{-}$
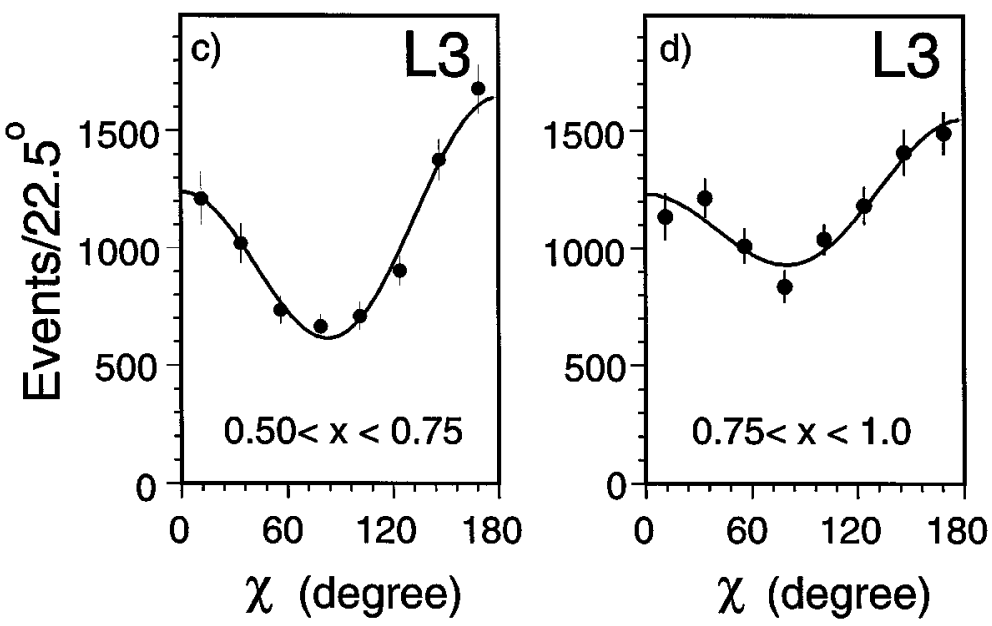

Fig. 7. The $\mu^{-}$azimuthal angle distribution for $-0.7 \leq z \leq 0.7$ in four intervals of $x$. The data are corrected for selection efficiency. The sample with $z<0$ is added to the sample with $z>0$ using the transformation $\chi \rightarrow \pi-\chi$. The curves show the function given in Eq. (7), with the value of $R_{X}$ and $R_{2 \chi}$ determined by a fit to the data in each $x$ interval (see Table 2).

\subsection{Determination of $F_{2}^{\gamma}$}

The structure function $F_{2}^{\gamma}$, as defined in Eq. (1), is extracted from the differential cross section, extrapolated to the full phase space, with no cut on $W_{\gamma \gamma}$ :

$$
F_{2}^{\gamma}\left(x, Q^{2}\right)=\mathscr{W}\left(x, Q^{2}\right) \frac{d^{2} \sigma}{d x d Q^{2}} .
$$

The weight $\mathscr{W}\left(x, Q^{2}\right)$ is calculated analytically using Monte Carlo events, for each bin of $x$ and $Q^{2}$, integrating the differential weight $\tilde{\mathscr{F}}$ over the kinematically allowed range of the scaled target photon energy $x_{2}$ :

$$
\frac{1}{\tilde{\mathscr{W}}\left(x, Q^{2}, x_{2}\right)}=K\left(x, Q^{2}, x_{2}\right)=\frac{8 \pi \alpha^{2}}{Q^{2}} \frac{x_{2}}{x}\left\{\left(\frac{s+Q^{2}}{W_{\gamma \gamma}^{2}+Q^{2}}\right) x_{2}-1\right\} \frac{d n\left(x_{2}\right)}{d x_{2}},
$$


where $d n\left(x_{2}\right) / d x_{2}$ is the target photon flux defined in Eq. (2). After changing variables from $Q^{2}, x, x_{2}$ to $Q^{2}, x, x_{1}$, for each Monte Carlo event we calculate the average value

$$
\mathscr{W}\left(x, Q^{2}\right)=\left\langle\frac{1}{K\left(x, Q^{2}, x_{1}\right)}\right\rangle=\int_{x_{1}^{\min }}^{x_{1}^{\max }} \frac{d x_{1}}{\left(x_{1}^{\max }-x_{1}^{\min }\right) K\left(x, Q^{2}, x_{1}\right)} .
$$

The kinematically allowed limits on $x_{1}$ are

$$
x_{1}^{\max }=\min \left\{1-\frac{E_{0}}{E_{\text {beam }}}, 1-\frac{Q^{2}}{s \sin ^{2}\left(\theta_{1}^{\max } / 2\right)}\right\}, \quad x_{1}^{\min }=\max \left\{\frac{W_{\gamma \gamma}^{2}}{s}, 1-\frac{Q^{2}}{s \sin ^{2}\left(\theta_{1}^{\max } / 2\right)}\right\}
$$

where $E_{0}$ is the minimum allowed energy of the tagged electron and $s=4 E_{\text {beam }}^{2}$.

The values of $F_{2}^{\gamma}$, thus obtained for the di-muon channel, are shown in Fig. 4. Assuming the target photon to be real $\left(t^{2}=0\right)$, the QED predictions are significantly higher than the measured values: $\chi^{2} / d o f=107 / 10$, corresponding to a probability $<10^{-4}$. Eq. (3), fitted to the data, gives a mean value for the virtuality $\left\langle t^{2}\right\rangle=0.033 \pm 0.005 \mathrm{GeV}^{2}$ with a probability of $8 \%$. The fitted value of the virtuality is in excellent agreement with the value of $0.034 \pm 0.002 \mathrm{GeV}^{2}$ calculated by the Monte Carlo including radiative corrections [17]. Without radiative corrections the expected mean virtuality of the target photon is $\left\langle t^{2}\right\rangle=0.024 \pm 0.003$, two standard deviations below the measurement. This measurement demonstrates that the correction due to the target photon virtuality cannot be neglected in studies of hadronic structure functions of the photon. The theoretical parametrisations of parton density functions must include also the dependence of the target photon virtuality, as underlined in Ref. [20].

Table 2

The parameters $R_{\chi}$ and $R_{2 \chi}$, obtained by a fit of the function of Eq. (7) to the azimuthal angle, $\chi$, distributions, are given for different $x$ and $z$ ranges. The first error is statistical and the second is systematic. The correction factors $C_{R_{\chi}}$ and $C_{R_{2}}$, used to extrapolate $R_{\chi}$ and $R_{2 \chi}$ to the full $z$ range, are also given. In the last four raws, $F_{2}^{\gamma}, F_{A}^{\gamma}$ and $F_{B}^{\gamma}$ are given for the full $\mathrm{z}$ range, with the statistical and systematic

\begin{tabular}{|c|c|c|c|}
\hline$\Delta x$ & $\Delta z$ & $R_{\chi}$ & $C_{R_{\chi}}$ \\
\hline $0-0.25$ & $0-0.7$ & $0.30 \pm 0.06 \pm 0.06$ & 0.6118 \\
\hline $0.25-0.5$ & $0-0.7$ & $0.10 \pm 0.07 \pm 0.05$ & 1.0185 \\
\hline $0.75-1$ & $0-0.7$ & $-0.20 \pm 0.07 \pm 0.05$ & 1.2089 \\
\hline $0-0.25$ & $-0.7-0$ & $-0.22 \pm 0.07 \pm 0.05$ & 0.6118 \\
\hline $0.75-1$ & $-0.7-0$ & $0.19 \pm 0.08 \pm 0.08$ & 1.2089 \\
\hline$\Delta x$ & $\Delta z$ & $R_{2 \chi}$ & $C_{R_{2 \chi}}$ \\
\hline $0-0.25$ & $-0.7-0.7$ & $0.24 \pm 0.06 \pm 0.06$ & 0.1933 \\
\hline $0.25-0.5$ & $-0.7-0.7$ & $0.35 \pm 0.06 \pm 0.12$ & 0.3184 \\
\hline $0-0.25$ & $0.090 \pm 0.008$ & $-0.007 \pm 0.012$ & $0.008 \pm 0.010$ \\
\hline $0.25-0.5$ & $0.405 \pm 0.016$ & $-0.018 \pm 0.016$ & $0.090 \pm 0.021$ \\
\hline $0.50-0.75$ & $0.597 \pm 0.020$ & $0.063 \pm 0.026$ & $0.168 \pm 0.040$ \\
\hline $0.75-1$ & $0.731 \pm 0.032$ & $0.087 \pm 0.031$ & $0.089 \pm 0.045$ \\
\hline
\end{tabular}
errors added in quadrature 


\subsection{Azimuthal correlations and determination of $F_{A}^{\gamma}$ and $F_{B}^{\gamma}$}

In Fig. 5 the generated distribution of $z=\cos \theta *$ is presented. It increases rapidly towards $z= \pm 1$. Also plotted in Fig. 5 is the selection efficiency, i.e. the ratio of selected events, after the full simulation and the analysis cuts, over the generated events. The selection efficiency drops to zero at $z= \pm 1$, due to the limited acceptance at small polar angle. The events in the central region, where the acceptance is high and uniform, are the most sensitive to the interference of the transverse and longitudinal photon amplitudes. The azimuthal correlation measurement is therefore restricted to $|z| \leq 0.7$. The measured angular distributions are well reproduced by the Monte Carlo simulation [13]. The data are corrected for acceptance and efficiency over a two-dimensional grid in the variables $z$ and $\chi$. Several correction methods are used to extract the azimuthal distributions, taking into account detector acceptance and resolution effects: a bin-by-bin correction using the ratio of reconstructed events over generated events and two unfolding algorithms [21,22]. The unfolding of Ref. [21] is used in the analysis. The $\chi$ distributions are fitted using a function of the form

$$
\frac{d N}{d \chi}=C\left[1+R_{\chi} \cos \chi+R_{2 \chi} \cos 2 \chi\right],
$$

with $R_{\chi}, R_{2 \chi}$ and $C$ let as free parameters. From Eqs. (4) and (5), it follows that $C \propto F_{2}^{\gamma}, R_{\chi} \propto F_{A}^{\gamma} / F_{2}^{\gamma}$ and $R_{2 \chi} \propto F_{B}^{\gamma} / F_{2}^{\gamma}$. The $x$ range is subdivided in four intervals. The coefficient $R_{2 \chi}$ is symmetric in $z$ and is determined by a fit over the range $-0.7<z<0.7$, where $R_{\chi}$ vanishes. The fit result, superimposed to the data, is shown in Fig. 6 . Since $F_{A}^{\gamma}$ is antisymmetric in $z$, the $x$ dependence of $R_{\chi}$ is studied in the ranges $-0.7<z<0$ and $0<z<0.7$. In Fig. 7 the sample with $z<0$ is added to the sample with $z>0$, using the transformation $\chi \rightarrow \pi-\chi$. The fit results of $R_{\chi}$ and $R_{2 \chi}$ are given in Table 2 with statistical and systematic errors.

Several sources of systematic errors on the measurement of $R_{\chi}$ and $R_{2 \chi}$ are taken into account. The effects of detector resolution on the variables $Q^{2}, x, z$ and $\chi$ are tested by Monte Carlo simulations, varying each variable in turn, according to its resolution. This leads to a systematic error of $8 \%$ on $R_{\chi}$ and $R_{2 \chi}$. The muon

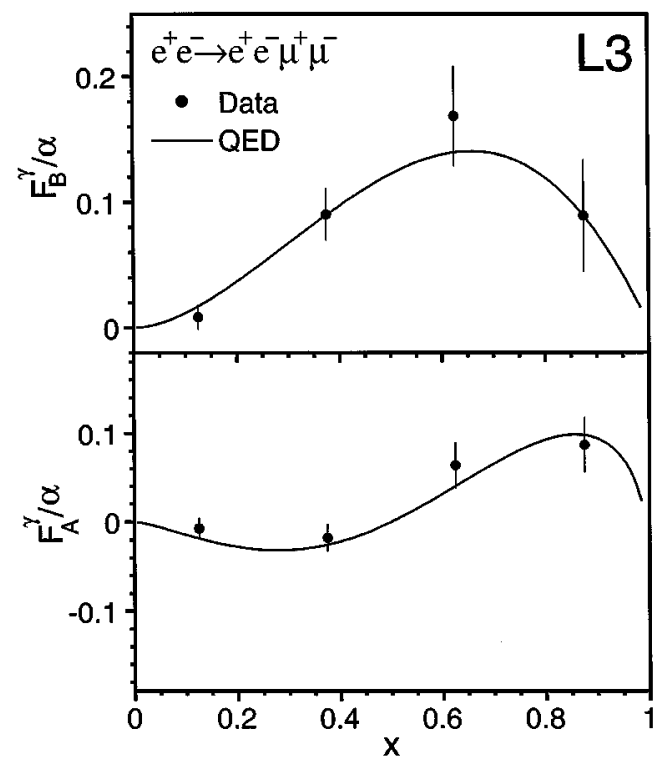

Fig. 8. The leptonic structure functions $F_{b}^{\gamma}$ and $F_{A}^{\gamma}$ as extracted from the $R_{\chi}, R_{2 \chi}$ and $F_{2}^{\gamma}$ measurements as function of $x$. The QED predictions from Eq. (5) (see text) are superimposed on the data. The structure function $F_{A}^{\gamma}$ is the weighted average between $F_{A}^{\gamma}(z>0)$ and $-F_{A}^{\gamma}(z<0)$. The statistical and systematic errors are added in quadrature. 
momentum resolution produces a systematic uncertainty of $12 \%$. By comparing predictions of the generators [14] and [17], a systematic error of $4 \%$ is assigned to the effect of radiative corrections. The effects of cuts on $z$ and $x$ contribute less than $3 \%$. From the average deviation between the results obtained with the three different unfolding methods used to correct the data, systematic errors of $1 \%$ on $R_{X}$ and $13 \%$ on $R_{2 X}$ are estimated.

To determine $F_{A}^{\gamma}$ and $F_{B}^{\gamma}$, the measurement is extrapolated to the full $z$ range. A set of correction factors $C_{R_{\chi}}$ and $C_{R_{2 \chi}}$ are calculated by numerical integration and reported in Table 2. The structure functions $F_{A}^{\gamma}$ and $F_{B}^{\gamma}$ are extracted as:

$$
F_{A}^{\gamma}=\frac{1}{2} C_{R_{\chi}} R_{\chi} F_{2}^{\gamma}, \quad F_{B}^{\gamma}=2 C_{R_{2 \chi}} R_{2 \chi} F_{2}^{\gamma} .
$$

Their values are given in Table 2 and shown in Fig. 8 together with the QED expectations. Good agreement is found in shape and absolute value. These results are the first measurements of the dependence of the structure functions $F_{A}^{\gamma}$ and $F_{B}^{\gamma}$ on the scaling variable $x$. The interference term of the longitudinal photon present in the structure function $F_{A}^{\gamma}$ is small, as expected, but measurable.

\section{Conclusions}

We have measured the differential cross sections of the single tag two-photon reaction $\mathrm{e}^{+} \mathrm{e}^{-} \rightarrow \mathrm{e}^{+} \mathrm{e}^{-} \mathrm{e}^{+} \mathrm{e}^{-}$ and $\mathrm{e}^{+} \mathrm{e}^{-} \rightarrow \mathrm{e}^{+} \mathrm{e}^{-} \mu^{+} \mu^{-}$at $\sqrt{s} \simeq 91 \mathrm{GeV}$, using data collected by the L3 detector at LEP during the years 1991 to 1994 . The data are in good agreement with QED predictions.

In the channel $\mathrm{e}^{+} \mathrm{e}^{-} \rightarrow \mathrm{e}^{+} \mathrm{e}^{-} \mu^{+} \mu^{-}$, the leptonic structure function $F_{2}^{\gamma}$ is extracted from the differential cross section $d^{2} \sigma / d x d Q^{2}$ in the $Q^{2}$ range $1.4 \mathrm{GeV}^{2}<Q^{2}<7.6 \mathrm{GeV}^{2}$.

By fitting the data, the mean value of the target photon virtuality is found to be $\left\langle t^{2}\right\rangle=0.033 \pm 0.005 \mathrm{GeV}^{2}$, in good agreement with QED predictions. Neglecting initial state radiative corrections the theory expectations are two standard deviations below the measurement.

The first measurement of the $F_{A}^{\gamma}$ and $F_{B}^{\gamma}$ leptonic structure functions is obtained by studying the azimuthal angle distribution of the $\mu^{-}$in the $\gamma \gamma$ centre-of-mass system. Both structure functions originate from interference terms of the scattering amplitudes. The $x$ dependence of the interference terms, as predicted by $\mathrm{QED}$, is indeed observed. This measurement establishes the presence of a longitudinal photon component in the single tag two-photon reaction.

\section{Acknowledgements}

We express our gratitude to the CERN accelerator divisions for the excellent performance of the LEP machine. We acknowledge with appreciation the effort of all engineers, technicians and support staff who have participated in the construction and maintenance of this experiment. We wish to thank G. d'Agostini, F.A. Berends and G.A. Schuler for useful discussions.

\section{References}

[1] V.M. Budnev, I.F. Ginzburg, G.V. Meledin, V.G. Serbo, Phys. Rep. C 15 (1974) 181.

[2] L3 Collaboration, M. Acciarri et al., Phys. Lett. B 407 (1997) 341.

[3] OPAL Collaboration, R. Ackers et a1., Z. Phys. C 60 (1993) 593.

[4] DELPHI Collaboration, P. Abreu et al., Z. Phys. C 96 (1994) 199.

[5] OPAL Collaboration, K. Ackerstaff et al., Z. Phys. C 74 (1997) 49.

[6] CELLO Collaboration, H.J. Behrend et al., Phys. Lett. B 126 (1983) 384. 
[7] P. Kessler, in: D.J. Miller, S.L. Cartwright, V. Khoze (Eds.), Proceedings of Photon'95, Sheffield U.K., 8-13 April 1995, World Scientific, Singapore, 1995, p. 281.

[8] J.H. Field, in: D.J. Miller, S.L. Cartwright, V. Khoze (Eds.), Proceedings of Photon'95, Sheffield U.K., 8-13 April 1995, World Scientific, Singapore, 1995 , p. 485.

[9] C. Peterson, P. Zerwas, T.F. Walsh, Nucl. Phys. B 229 (1983) 301, Note that in Eqs. (21) a factor $\frac{1}{2}$ is missing in the first equation, a factor $x$ in the third one.

[10] L3 Collaboration, B. Adeva et al., Nucl. Inst. Meth. A 289 (1990) 35.

[11] L3 Collaboration, B. Adeva et al., Nucl. Inst. Meth. A 381 (1996) 236.

[12] R. Bizzarri et al., Nucl. Inst. Meth. A 317 (1992) 463.

[13] G. Susinno, Ph.D. thesis 2961, Université de Genève, Switzerland, 1997.

[14] F.A. Berends, P.H. Daverfeldt, R. Kleiss, Nucl. Phys. B 253 (1985) 441; Comp. Phys. Comm. 40 (1986) 285.

[15] J. Vermaseren, Nucl. Phys. B 229 (1983) 347.

[16] R. Brun et al., GEANT 3, CERN DD/EE/84-1 (Revised), September 1987.

[17] F.A. Berends, P.H. Daverfeldt, R. Kleiss, Nucl. Phys. B 253 (1985) 421; Comp. Phys. Comm. 40 (1986) 271.

[18] KORALZ version 4.0 is used, S. Jadach, B.F.L. Ward, Z. Was, Comp. Phys. Comm. 79 (1994) 503.

[19] PYTHIA version 5.7 is used, T. Sjöstrand, Comp. Phys. Comm. 82 (1994) 74.

[20] G. Schuler, T. Sjöstrand, Phys. Lett. B 376 (1996) 193.

[21] G. D'Agostini, Nucl. Inst. Meth. A 362 (1995) 487.

[22] A. Höcker, V. Kertvelishvili, Nucl. Inst. Meth. A 372 (1996) 469. 